\title{
Primary hypertrophic osteoarthropathy due to a novel SLCO2A1 mutation masquerading as acromegaly
}

\author{
Ruth Mangupli', Adrian F Daly², Elvia Cuauro', Paul Camperos', Jaime Krivoy \\ and Albert Beckers ${ }^{2}$
}

1Department of Neurosurgery, Section of Neuroendocrinology, Hospital Universitario de Caracas, Caracas, Venezuela and 2Department of Endocrinology, Centre Hospitalier Universitaire de Liège, University of Liège, Liège, Belgium

\author{
Correspondence \\ should be addressed \\ to R Mangupli or A Beckers \\ Email \\ nenhuc@gmail.com or \\ albert.beckers@chu.ulg.ac.be
}

\section{Summary}

A 20-year-old man with an 8-year history of progressive enlargement of his hands and feet, coarsening facial features, painful joints and thickened, oily skin was referred for investigation of acromegaly. On examination, the subject was of normal height and weight. He had markedly increased skin thickness around the forehead, eyelids and scalp with redundant skin folds. Bilateral painful knee swelling was accompanied by enlargement of the extremities, and his fingers were markedly clubbed. Routine hematological, biochemical and hormonal blood tests, including GH and IGF-1 were normal. The clinical picture suggested primary hypertrophic osteoarthropathy (PHOA) rather than acromegaly and radiological studies were supportive of this, demonstrating increased subperiosteal bone formation and increased bone density and cortical thickening. There was widespread joint disease, with narrowing of joint spaces, whereas the knees demonstrated effusions and calcification. A skull X-ray revealed calvarial hyperostosis and a normal sellar outline. Family history was negative. Genetic studies were performed on peripheral blood leukocyte DNA for mutations in the two genes associated with PHOA, 15-hydroxyprostaglandin dehydrogenase (HPGD; OMIM: 601688) and solute carrier organic anion transporter family member 2A1 (SLCO2A1; OMIM: 601460). The sequence of HPGD was normal, whereas the subject was homozygous for a novel pathological variant in SLCO2A1, c.830delT, that predicted a frameshift and early protein truncation ( $p$. Phe277Serfs*8). PHOA, also known as pachydermoperiostosis, is a rare entity caused by abnormal prostaglandin E2 metabolism, and both HPGD and SLCO2A1 are necessary for normal prostaglandin E2 handling. High prostaglandin levels lead to bone formation and resorption and connective tissue inflammation causing arthropathy, in addition to soft tissue swelling.

\section{Learning points:}

- The differential diagnosis of enlarged extremities, coarsened facial features, skin changes and increased sweating in suspected acromegaly is quite limited and primary hypertrophic osteoarthropathy (PHOA) is one of the few conditions that can mimic acromegaly at presentation.

- PHOA is not associated with abnormalities in GH and IGF-1 secretion and can be readily differentiated from acromegaly by hormonal testing.

- Clubbing in the setting of diffuse enlargement of joints and extremities in addition to skin changes should alert the physician to the possibility of PHOA, as clubbing is not a usual feature of acromegaly. Underlying causes of secondary hypertrophic osteoarthroapthy (e.g. bronchial neoplasia) should be considered.

- PHOA is a very rare condition caused by abnormalities in prostaglandin metabolism and has two known genetic causes (HPGD and SLCO2A1 mutations). 
- SLCO2A1 gene mutations lead usually to autosomal recessive PHOA; fewer than 50 SLCO2A1 mutations have been described to date and the current case is only the second in a Hispanic patient.

- Treatment of primary hypertrophic osteoarthropathy is focused on the management of joint pain usually in the form of non-steroidal anti-inflammatory drug therapy.

\section{Background}

The current case illustrates well the potential for PHOA to present as a queried case of acromegaly. The presentation at a community clinic with a gradual onset of increased size of hands and feet, facial coarsening, increased sweating and seborrhea led to a referral to a specialist endocrinology department to rule out acromegaly. The differential diagnosis of acromegaly is quite limited, so it is important to be aware of PHOA as a rare differential. The young male patient had the full clinical picture of PHOA (pachyderma, clubbing and periostosis), and he was homozygous for a novel pathological change in the SLCO2A1 gene. This mutation has not been reported previously and is only the second SLCO2A1 mutation reported in Latin America.

\section{Case presentation}

A 20-year-old Venezuelan male patient was referred for investigation of a possible diagnosis of acromegaly. He was of normal height $(170 \mathrm{~cm})$ and weight $(62 \mathrm{~kg})$. Beginning at the age of 12 years, he had progressive enlargement of his hands and feet and painful swelling of his knees and ankles. There was no family history of similar complaints. On examination, the patient had coarse facial features, thickened eyelids and partial blepharoptosis. Extensive dark, oily redundant skin folds were present on the forehead and scalp with prominent grooves consistent with cutis verticis gyrata (Fig. 1). His hands and feet were swollen, and his hands were markedly clubbed (Fig. 2). His main clinical complaint was knee pain, due to extensive swelling and effusions bilaterally.

\section{Investigation}

Routine hematological and biochemical blood tests were within normal limits. His GH, IGF-1 and thyroid function tests were also normal, indicating that acromegaly was not the cause of his clinical features. Urinary testing for prostaglandins and metabolites was not available.
Radiologic studies of his limbs demonstrated subperiostial bone formation with bone density and cortical thickening (Fig. 3). Hand radiographs revealed similar subperiosteal bone formation, phalangeal thickening and decreased joint spaces at the carpus (Fig. 4). The knees showed bony excrescences in the patellae and involvement of the proximal tibia and distal femur with calcification and signs of effusion. A lateral radiograph of the skull demonstrated a normal sella with hyperostosis of the skull bones (Fig. 5). The patient had no signs of pulmonary disease, and PHOA was diagnosed. Given the known genetic causes of PHOA, the patient underwent sequencing of the hydroxyprostaglandin dehydrogenase

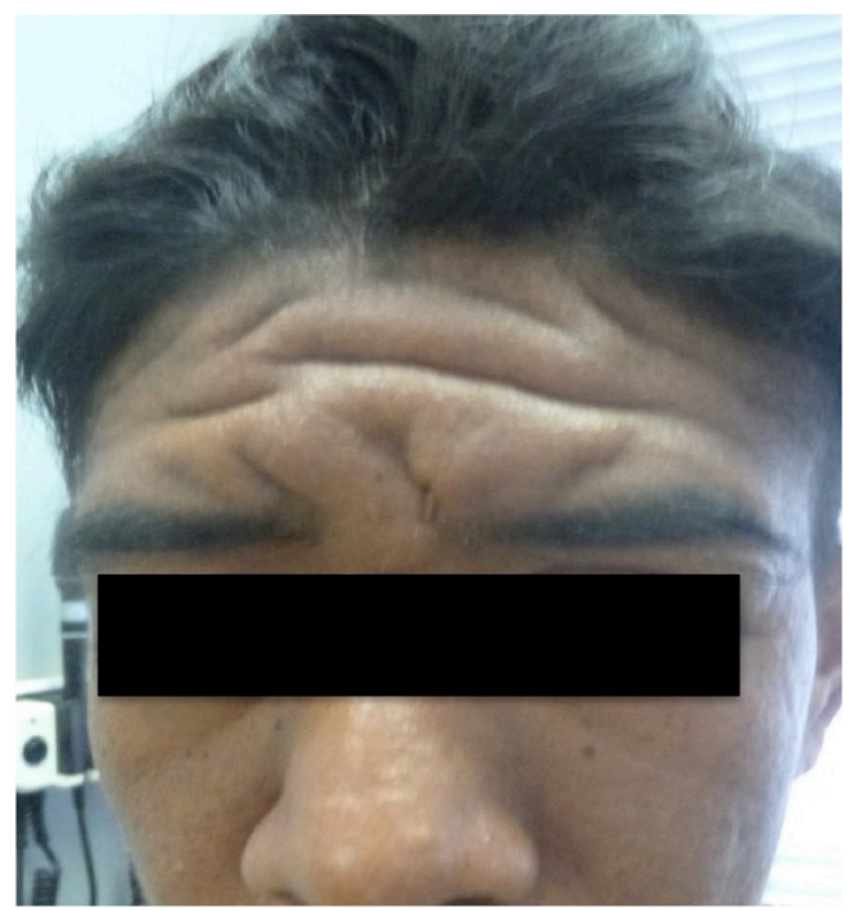

Figure 1

Facial changes in primary hypertrophic osteoarthropathy demonstrating thickened oily skin and excessive folding of the skin of the forehead (cutis verticis gyrata). 


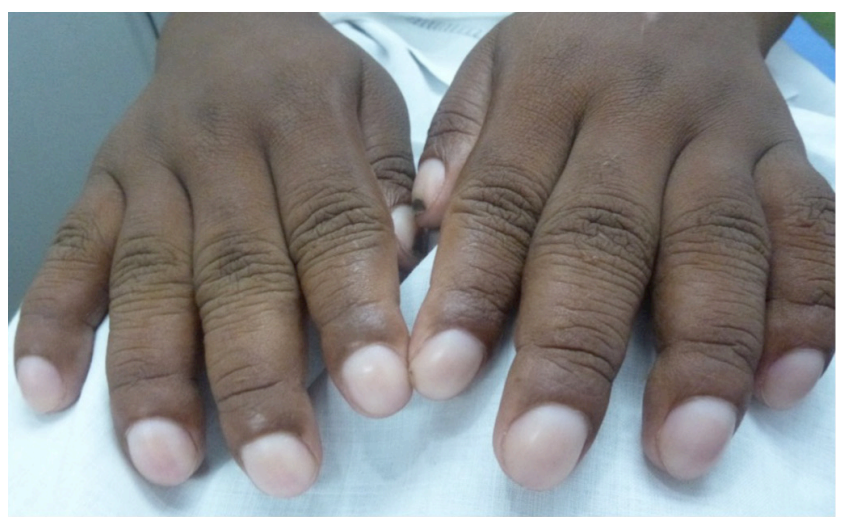

Figure 2

Digital clubbing in primary hypertropic osteoarthropathy.

(HPGD) and solute carrier organic anion transporter family member 2A1 (SLCO2A1) genes.

\section{Treatment}

Once a diagnosis of acromegaly had been discounted and the multifocal bone and joint disease diagnosed, he was treated with oral bisphosphonate therapy and regular non-steroidal anti-inflammatory drugs to reduce pain and inflammation.
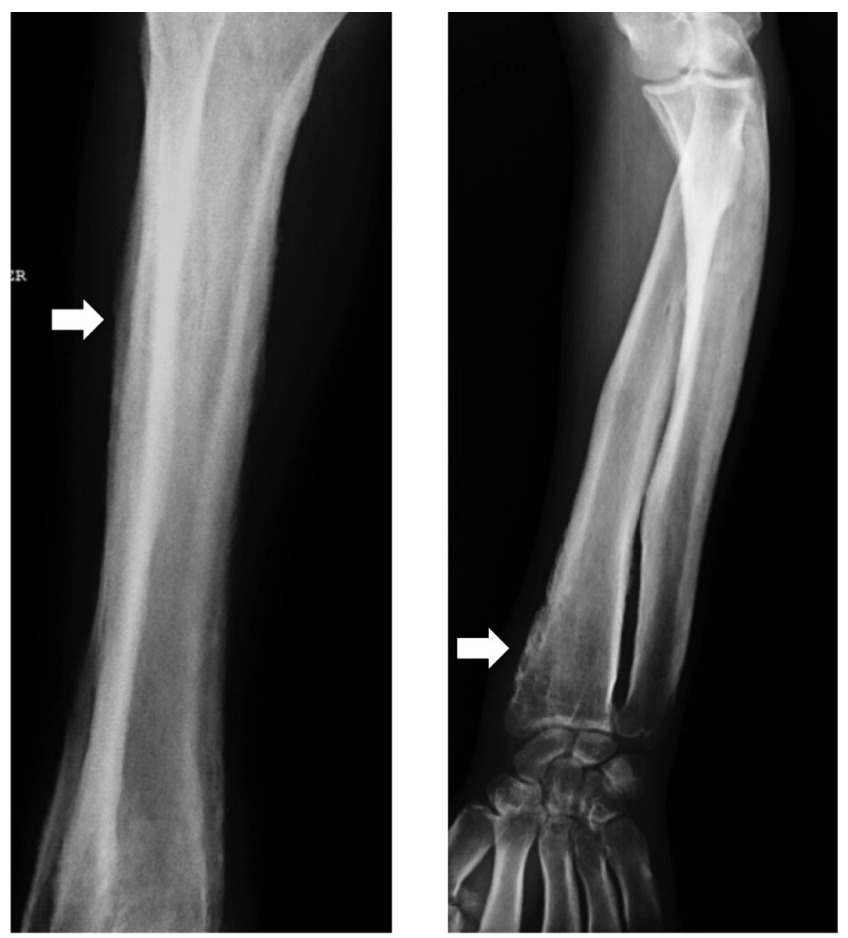

Figure 3

Periosteal new bone formation (periostosis) in the tibia and radius (white arrows) with associated cortical thickening.

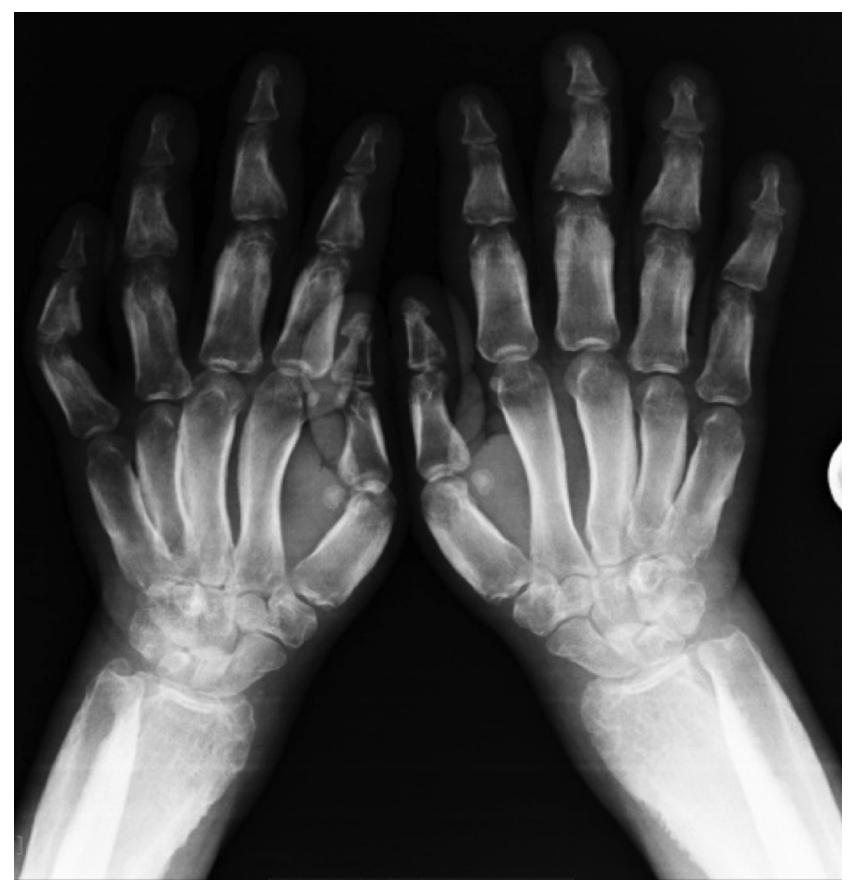

Figure 4

Typical radiological features of the distal upper limb and hands of a patient with primary hypertrophic osteoarthropathy showing increased density of the carpal, metacarpal and phalangeal bones with reduction in joint spaces and soft tissue swelling.

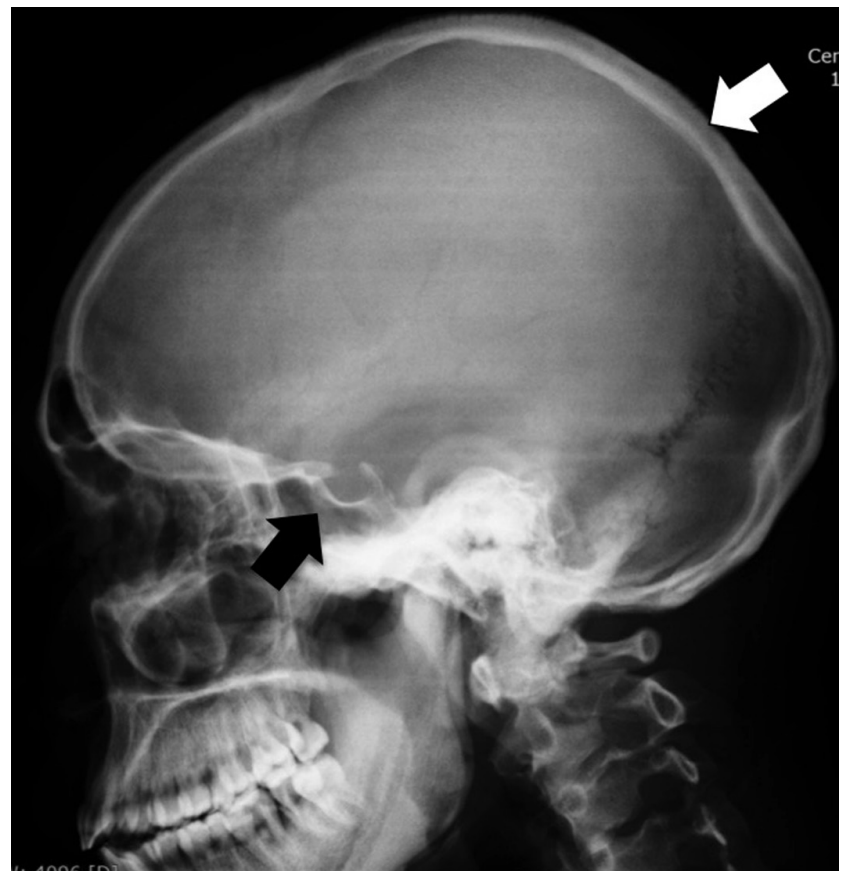

Figure 5

Lateral radiograph of the cranium showing a normal sella turcica (black arrow) and cranial hyperostosis (white arrow). 


\section{Outcome and follow-up}

Pain and joint swelling improved substantially after the initiation of non-steroidal anti-inflammatory therapy and bisphosphonate treatment. After two years of follow-up, the joint swelling and discomfort remains controlled, but there was no improvement in the skin disorder and established bone disease. The subject is scheduled for plastic surgery for reduction of facial skin folds and blepharoplasty for relief of his blepharoptosis.

\section{Discussion}

PHOA or pachydermoperiostosis is characterized by pachyderma, digital clubbing and periostosis $(1,2)$. It has an insidious onset, usually beginning in adolescence and presents with progressively worsening joint pain, enlargement of the extremities and coarsening facial features. The current case exemplifies this classical presentation and also the issue of being potentially mistaken for acromegaly. The patient was initially seen in a regional clinic and the presentation of facial changes and enlarged extremities led to his referral to exclude acromegaly. However, acromegaly was readily excluded by hormonal testing, whereas the presence of clubbing without underlying pulmonary, hepatic or malignant disease in a young individual pointed strongly to PHOA. This was supported by the radiological findings, with evidence of subperiosteal new bone formation, cortical thickening, epiphyseal widening and joint space narrowing.

PHOA is a rare disorder that was first described by Friedreich in the Hafner brothers in 1868 (3). In total, only a few hundred cases of primary hypertrophic osteoarthropathy have been reported in the literature $(1,2)$. The presentation can vary from the complete clinical picture seen in the current case to other forms affecting only the skeleton or the skin. After onset and worsening of clinical signs during adolescence and young adulthood, the clinical features of the disease tend to stabilize.

Most patients with this disease have autosomal recessive inheritance of inactivating mutations in either the HPGD or the SLCO2A1 gene $(4,5)$. The current case had a homozygous frameshift and early truncating mutation of the SLCO2A1 gene that has not been reported previously. This gene encodes a prostaglandin transporter protein, and patients with such mutations have increased levels of prostaglandin $\mathrm{E} 2$, which is in keeping with the proposed etiology of PHOA as a prostaglandin metabolism disorder. A recent overview of SLCO2A1 mutations in primary hypertrophic osteoarthropathy kindreds reported that less than 50 mutations have been identified in the clinical setting to date (6). Despite the genetic features in the current case, no other affected family members were identified.

The skeletal features in the current case were typical of those seen in the disease showing, subperiosteal new bone formation, cortical thickening and narrowing of joint spaces. Resorption of bone of the distal phalanges (acro-osteolysis) and ossification of inter-osseous membranes and ligaments can also be seen. Management is based on reduction of inflammation via non-steroidal anti-inflammatory drugs and bisphosphonates, whereas cosmesis can be improved with plastic surgery to remove excessive skin folds on the forehead and scalp and to address blepharoptosis, as is envisaged in the current case (7).

\section{Declaration of interest}

The authors declare that there is no conflict of interest that could be perceived as prejudicing the impartiality of the research reported.

\section{Funding}

The work to establish the genetic analysis was supported by a grant from the Fonds d'Investissement pour la Recherche (FIRS), 2016 from the Centre Hospitalier Unversitaire de Liège, Belgium (to Prof. Albert Beckers).

\section{Patient consent}

Written informed consent was obtained from the patient for the studies undertaken to establish the diagnosis and for publication of the current case report.

\section{Author contribution statement}

The patient was investigated, diagnosed and treated by Ruth Mangupli, Elvia Cuauro, Paul Camperos and Jaime Krivoy. The genetic diagnosis was established by Albert Beckers and Adrian F Daly. The study was written by Ruth Mangupli and Adrian F Daly.

\section{References}

1 Castori M, Sinibaldi L, Mingarelli R, Lachman RS, Rimoin DL \& Dallapiccola B 2005 Pachydermoperiostosis: an update. Clinical Genetics 68 477-486. (doi:10.1111/j.1399-0004.2005.00533.x)

2 Carneiro M, Hernandez D, Montes de Oca I, Sanchez J, Castillo E, Serrao M \& Gianonni M 1998 Paquidermoperiostosis (Osteoartropatia Hipertrófica Primaria). Presentación de un caso y revisión de la literatura. Gaceta Medica Caracas 106 540-548. 
Endocrinology

Diabetes \& Metabolism

CASE REPORTS
R Mangupli and others

PHOA masquerading as acromegaly
ID: 17-0013; April 2017

DOI: 10.1530/EDM-17-0013
3 Friedreich N 1868 Hyperostose des gessamten Skelletes. Archiv für Pathologische Anatomie und Physiologie und für Klinische Medicin 4383. (doi:10.1007/bf02117271)

4 Uppal S, Diggle CP, Carr IM, Fishwick CW, Ahmed M, Ibrahim GH, Helliwell PS, Latos-Bieleńska A, Phillips SE, Markham AF, et al. 2008 Mutations in 15-hydroxyprostaglandin dehydrogenase cause primary hypertrophic osteoarthropathy. Nature Genetics 40 789-793. (doi:10.1038/ng.153)

5 Zhang Z, Xia W, He J, Zhang Z, Ke Y, Yue H, Wang C, Zhang H, Gu J, $\mathrm{Hu}$ W, et al. 2012 Exome sequencing identifies SLCO2A1 mutations as a cause of primary hypertrophic osteoarthropathy. American Journal of Human Genetics 90 125-132. (doi:10.1016/j.ajhg.2011.11.019)

6 Lee S, Park SY, Kwon HJ, Lee CH, Kim OH \& Rhee Y 2016

Identification of the mutations in the prostaglandin transporter gene, SLCO2A1 and clinical characterization in Korean patients with pachydermoperiostosis. Journal of Korean Medical Science 31 735-742. (doi:10.3346/jkms.2016.31.5.735)

7 Liu CY \& Zhang YF 2014 Pachydermoperiostosis images in clinical medicine. New England Journal of Medicine 370 20. (doi:10.1056/ NEJMicm1310638)

Received in final form 16 March 2017

Accepted 24 March 2017 